\title{
Tratamento nutricional em pacientes com insuficiência cardíaca
}

\author{
Nutritional treatment for \\ heart failure patients
}

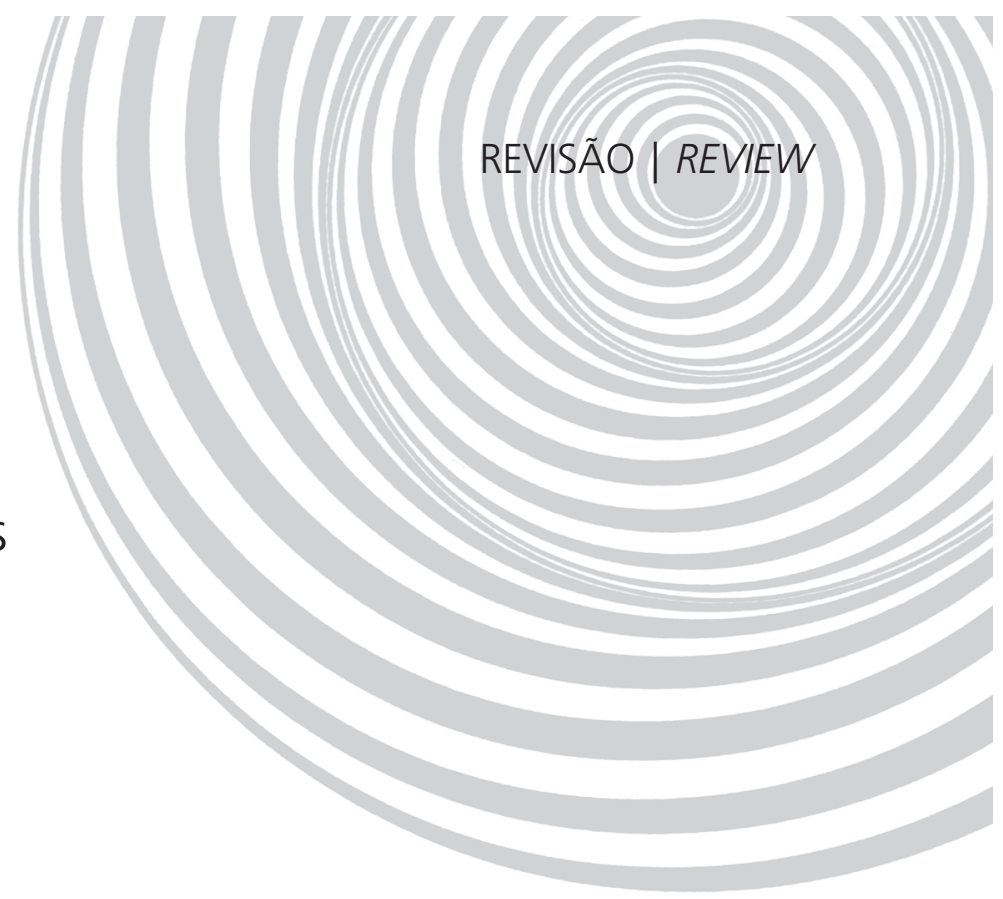

Viviane SAHADE ${ }^{1}$

Vanessa dos Santos Pereira MONTERA 2,3

RE S U M O

A insuficiência cardíaca é um problema grave e crescente de saúde pública em todo o mundo, sendo a via final comum da maioria das cardiopatias. Apesar dos recentes avanços da medicina, pouca atenção vem sendo dada ao tratamento não farmacológico, em particular à terapia nutricional. A evolução clínica dos pacientes com insuficiência cardíaca, via de regra, caminha para quadros variáveis de desnutrição, de causa multifatorial e que não está necessariamente relacionada ao índice de massa corporal. Assim, o objetivo deste trabalho foi revisar a literatura na busca por evidências sobre a composição adequada da dieta no tratamento de pacientes com insuficiência cardíaca. Realizou-se uma revisão sistemática nas bases de dados Medline, Pubmed, Lilacs, SciELO e dos comitês nacionais e internacionais de saúde, dos artigos publicados no período compreendido entre 1981 e 2006. Observou-se que existem poucos estudos bem elaborados, sobre a adequação da dietoterapia na insuficiência cardíaca e que esta ainda é muito restrita ao controle do sal e de líquido, sendo dada pouca atenção à quantidade e à qualidade dos demais nutrientes. Existe uma crescente evidência de que a dieta é um fator importante no prognóstico e no tratamento desses pacientes, porém futuros estudos, incluindo ensaios clínicos com adequado número de participantes, se fazem necessários para que se possa prescrever, com segurança, suplementos nutricionais.

Termos de indexação: Desnutrição. Insuficiência cardíaca. Macronutrientes. Micronutrientes. Nutrição em saúde pública.

A B S TR A C T

Heart failure is a serious and increasing public health problem around the world, and is the common endpoint of most heart diseases. Despite recent medical advances, little attention has been given to the nonpharmacological treatments, specifically, to nutritional therapy. The clinical course of patients with heart

1 Universidade Federal da Bahia, Escola de Nutrição, Departamento de Ciências da Nutrição. Av. Araújo Pinho, 32, Canela, 40110-150, Salvador, BA, Brasil. Correspondência para/Correspondence to: V. SAHADE. E-mail: <vivianesahade@uol.com.br>.

2 Santa Casa da Misericórdia do Rio de Janeiro. Rio de Janeiro, RJ, Brasil.

${ }^{3}$ Mestranda, Universidade Federal Fluminense, Programa de Pós-Graduação em Ciências Cardiovasculares. Rio de Janeiro, RJ, Brasil. 
400 | V. SAHADE \& V.S.P. MONTERA

failure constantly progresses to varying degrees of malnutrition caused by different factors and not necessarily related to body mass index. Thus, the objective of this study was to search the literature for evidences of a proper diet composition for heart failure patients. The Medline, Pubmed, Lilacs and SciELO databases, and the national and international health committees were systematically searched for articles published between 1981 and 2006. There were few well-designed studies on proper diet therapies for heart failure patients. The ones reviewed focused on restricting table salt and liquids but gave little attention to the amount and quality of other nutrients. There is growing evidence that diet is an important factor in the prognosis and treatment of these patients. However, future studies, including clinical trials with adequate numbers of participants, are necessary if nutritional supplements are to be safely prescribed.

Indexing terms: Heart failure. Malnutrition. Micronutrient. Macronutrient. Nutrition, public health.

\section{N T R O D U ÇÃ O}

A insuficiência cardíaca (IC) é uma síndrome clínica ocasionada por uma anormalidade da função do coração em bombear e/ou em acomodar o retorno sangüíneo, não atendendo às necessidades de oxigênio dos tecidos ou apenas oferecendo um adequado débito cardíaco pelo aumento anormal das pressões de enchimento, deflagrando uma complexa resposta neuro-humoral e inflamatória.

A IC é um problema grave e crescente de saúde pública em todo o mundo, sendo a via final comum da maioria das cardiopatias. Apesar de os avanços científicos e tecnológicos e as melhores condições socioeconômicas terem possibilitado o aumento da longevidade da população geral e dos cardiopatas, tem-se registrado aumento da incidência de IC no mundo². Aproximadamente 23 milhões de pessoas são portadores dessa doença e dois milhões de novos casos são diagnosticados a cada ano ${ }^{3}$.

No Brasil não existem estudos epidemiológicos envolvendo a incidência de insuficiência cardíaca, porém, de acordo com outros países, pode-se estimar que até 6,4 milhões de brasileiros sofram dessa síndrome ${ }^{4}$. Segundo dados obtidos do Sistema Único de Saúde (SUS) ${ }^{5}$, do Ministério da Saúde, foram realizadas, nos primeiros sete meses do ano de 2003, 203893 internações por IC, o que corresponde a uma taxa de 68,7/1000 internações, com ocorrência de 14 mil óbitos.

A evolução clínica dos pacientes com IC, via de regra, caminha para quadros variáveis de desnutrição. Esta pode ocorrer devido à ingestão inadequada, ao metabolismo alterado, ao estado pró-inflamatório, ao aumento do estresse oxidativo e à maior perda de nutrientes, até mesmo pelas interações medicamentosas. A anorexia é conseqüência da redução da ingestão de nutrientes ou da associação das alterações absortivas e metabólicas (hipermetabolismo, hipóxia, aumento do gasto energético, inflamação). O edema das alças intestinais na insuficiência cardíaca pode ser responsável pela presença de náuseas, má absorção de lipídios, sensação de plenitude gástrica e de perdas protéicas ${ }^{6,7}$.

A presença da desnutrição constitui importante fator preditivo de redução de sobrevida nos pacientes com IC independentemente de variáveis importantes como idade, classe funcional e fração de ejeção ${ }^{8}$.

Apesar do histórico entrelaçamento entre desnutrição e insuficiência cardíaca, poucos são os estudos que discutem o papel da terapia nutricional no tratamento desses pacientes. A fim de que se possa ampliar a visão do papel do nutricionista no tratamento de pacientes com insuficiência cardíaca, até então muito restrita ao controle de sódio e líquido, o presente estudo teve como objetivo revisar a literatura na busca de evidências sobre a composição adequada da dieta no tratamento de pacientes com IC.

\section{MÉTODOS}

Realizou-se uma revisão da literatura de forma sistemática, nas bases de dados Medline, Pubmed, Lilacs, SciELO e dos comitês nacionais e internacionais de saúde, dos artigos publicados 
no período compreendido de 1981 a 2006. Na estratégia de busca, foram utilizadas as seguintes palavras-chave: nutrition, heart failure, malnutrition, micronutrient, macronutrient. Os artigos foram pré-selecionados a partir da leitura do resumo disponível. Foram incluídas publicações em português e em inglês com dados primários realizados em pacientes hospitalizados ou atendidos em ambulatórios. A busca bibliográfica, segundo a estratégia estabelecida, resultou em 90 artigos, sendo 60 na base PubMed e 30 nas bases Medline, Lilacs e SciELO. No entanto, foram excluídos: artigos com crianças e adolescentes (23), os estudos repetidos nas diferentes bases de dados (20), os repetidos quando as palavras-chave foram variadas (12), e os que não foram encontrados na íntegra (5). Ao final, foram selecionados 30 artigos para esta revisão e 9 publicações oriundas de comitês nacionais e internacionais de saúde.

\section{RESULTADOSE DISCUSSÃ O}

\section{Valor energético da dieta}

Pacientes com insuficiência cardíaca apresentam alteração do balanço anabolismo/catabolismo resultante de modificações neurohormonais marcadas pelo aumento dos níveis de fatores catabólicos (norepinefrina, epinefrina, angiotensina II, cortisol, citocinas inflamatórias e radicais livres) e pela resistência a hormônios anabólicos, como hormônio do crescimento $(\mathrm{GH})$ e insulina. Estas modificações contribuem para um aumento do gasto energético em repouso ${ }^{9}$.

Pacientes em classes funcionais III e IV ${ }^{10}$ apresentam aumento da taxa metabólica basal em torno de $18 \%$, em comparação com indivíduos saudáveis. $\mathrm{O}$ aumento das demandas energéticas pelo trabalho dos músculos respiratórios, pelo miocárdio hipertrofiado e pelo sistema hemopoiético contribui para o aumento da taxa metabólica basal ${ }^{11}$.

Associada ao estado hipercatabólico encontra-se a má absorção intestinal, favorecida pela congestão gastrintestinal. Estudos ${ }^{12,13}$ demonstram a relação entre enteropatias com perda de proteína e gordura, e um quadro de anorexia, relacionada a medicamentos, depressão, elevação dos níveis circulantes de citocinas e angiotensina II e alteração do paladar.

Com relação aos pacientes com sobrepeso e obesidade, a restrição energética é discutível. Um estudo ${ }^{14}$ concluiu que a obesidade e o sobrepeso são fortes preditores para o desenvolvimento da IC. Embora a obesidade cause anormalidades na função distólica e sistólica e predisponha a insuficiência cardíaca, diversos estudos demonstram que pacientes obesos com IC, paradoxalmente parecem ter um prognóstico clinico favorável ${ }^{15-17}$. Em uma pesquisa ${ }^{15}$ que estudou a relação da obesidade e mortalidade, foram analisados 1203 indivíduos, a maioria com classe funcional I, e observou-se que a elevação do Índice de Massa Corporal (IMC) estava associada a uma maior taxa de sobrevida. Quando se aplicou a análise multivariada, houve uma associação inversa entre IMC e mortalidade.

Nos pacientes idosos com sobrepeso, a perda voluntária de peso associa-se a maior risco de mortalidade. A ingestão de dieta hipoenergética $(<24 \mathrm{kcal} / \mathrm{kg} / \mathrm{dia}$ ) pode aumentar o desequilíbrio dos hormônios catabólicos e fazer com que ocorra maior perda de massa magra ${ }^{18}$.

Em um estudo ${ }^{18}$, a partir da análise da ingestão nutricional, foram estabelecidas recomendações energéticas para pacientes com IC clinicamente estáveis. Estas são de $28 \mathrm{kcal} / \mathrm{kg}$ para pacientes com estado nutricional adequado e $32 \mathrm{kcal} / \mathrm{kg}$ para pacientes nutricionalmente depletados. Para este cálculo deve-se levar em consideração o peso do paciente na ausência de retenção hídrica.

\section{Carboidratos}

Os carboidratos devem representar em torno de 50 a $60 \%$ do valor energético da dieta, evitando-se os carboidratos simples e dando 
preferência aos carboidratos de baixa carga glicêmica. O excesso de carboidratos, especialmente os de alta carga glicêmica, pode agravar o quadro de resistência à insulina, comumente percebido em pacientes com insuficiência cardíaca, o que representa mau prognóstico para estes. A insulina é um hormônio natriurético e a resistência a ela pode agravar a retenção de sódio e águaa,19,20.

A recomendação diária de fibra de 20 a $30 \mathrm{~g}$ previne a obstipação intestinal e o conseqüente esforço para evacuar, o qual deve ser evitado ${ }^{21}$. As fibras contribuem para a produção de ácidos graxos de cadeia curta que nutrem os enterócitos, favorecendo a redução da hiperpermeabilidade intestinal e, assim, diminuindo a passagem de moléculas capazes de ativar o sistema imune, o que aumentaria o estado pró-inflamatório ${ }^{21}$.

\section{Proteínas}

As necessidades protéicas para o adulto normal variam entre 0,8 a $1,0 \mathrm{~g} / \mathrm{kg}$ de peso corporal/dia. Estudos mais recentes em insuficiência cardíaca ${ }^{18}$, indicam necessidades protéicas de $1,1 \mathrm{~g} / \mathrm{kg} /$ dia para pacientes com estado nutricional adequado e de $1,5 \mathrm{~g} / \mathrm{kg} / \mathrm{dia}$ a $2,0 \mathrm{~g} / \mathrm{kg} /$ dia para os com depleção nutricional ou que apresentem perdas por nefropatia ou má absorção intestinal. Para ambos os casos deve ser considerado o peso livre de edemas. Com isso, a relação caloria/grama nitrogênio deve ficar entre 120 e 160, de preferência uma relação em torno de $150^{22}$.

Pacientes que desenvolvem insuficiência renal devem receber orientações individualizadas em relação às proteínas ${ }^{22}$.

\section{Lipídios}

Os lipídios complementam o valor energético total da dieta, não excedendo 30\% deste. Mais importante que a quantidade dos lipídios é a qualidade dos mesmos, principalmente se o paciente apresentar hiperlipidemia. Deve-se evitar o consumo de alimentos contendo gordura trans, reduzir a ingestão de gordura saturada e dar preferência às gorduras mono e polinsaturadas, com ênfase aos ácidos graxos da série ômega 3, que apresentam efeitos positivos em pacientes com insuficiência cardíaca sintomáticos 23,24 .

A má absorção de gordura ocorre em cerca de 1/3 dos pacientes com caquexia cardíaca e, na ocorrência de esteatorréia, indica-se a suplementação de triglicerídeos de cadeia média? .

\section{Restrição de sódio e líquido}

A quantidade de sódio oferecida na dieta depende da gravidade da insuficiência cardíaca. Um estudo ${ }^{25}$ com seguimento de 19 anos verificou a relação entre dieta sem restrição de sódio e a incidência de IC congestiva. Esse estudo envolveu 10362 indivíduos sem história de IC, com peso normal ou sobrepeso. Os resultados apontaram o alto consumo de sódio como um fator de risco independente para IC e hipertrofia ventricular esquerda nos indivíduos com sobrepeso.

No Brasil, a ingestão diária de cloreto de sódio situa-se em torno de 8 a 12g/dia, ultrapassando em mais de cinco vezes as necessidades diárias ${ }^{4}$. No paciente com insuficiência cardíaca severa, a ingestão de sódio deve ser, no máximo, de 2-3g/dia, podendo ser modificada de acordo com o sódio plasmático e a tolerância à dieta hipossódica ${ }^{26}$.

Além do controle do sal adicionado às preparações, deve-se orientar o paciente quanto aos alimentos com alto teor de sódio. O monitoramento do sódio sérico deve ser realizado sempre que possível, uma vez que a hiponatremia ocasiona vários efeitos adversos ${ }^{20,23,27}$.

O grande problema da dieta hipossódica é a baixa adesão, devido à palatabilidade dos alimentos. Um recente artigo de revisão demonstrou variações na taxa de não adesão à restrição de sódio de 50 a $88 \%{ }^{28}$. É necessário fornecer orientações e receitas com temperos e ervas naturais para serem usados nas preparações, pois 
somente com a educação nutricional a adesão à restrição sódica irá acontecer de maneira efetiva e eficaz.

A restrição hídrica nem sempre é necessária, irá depender da gravidade da insuficiência cardíaca. Nos pacientes com IC severa, nos quais a concentração de hormônio antidiurético circulante pode estar aumentada e a capacidade de eliminação de água prejudicada, a restrição hídrica é aconselhada para evitar a retenção hídrica e situações de hiponatremia nas quais a concentração de sódio plasmático atinja níveis inferiores a $130 \mathrm{mEq} / \mathrm{L}^{26}$.

Na prática diária, a quantidade máxima de 2,0L/dia é recomendada. No entanto, em pacientes com estado congestivo, a ingestão hídrica pode ser menor, devendo ser restringida de acordo com a superfície corporal, na busca de um balanço hídrico negativo inicial, até que se alcance um estado normovolêmico. O valor de restrição máxima pode atingir 600 a $700 \mathrm{ml} / \mathrm{m}^{2}$ da superfície corporal/dia ${ }^{26}$.

\section{Álcool}

O uso excessivo de bebida alcoólica deve ser desencorajado, em função de seus efeitos negativos sobre o sistema cardiovascular. O álcool reduz a contratibilidade miocárdica e pode causar arritmias. Alguns estudos ${ }^{29-31}$ demostraram que o consumo moderado de álcool, até 30g/dia para homens e $20 \mathrm{~g} / \mathrm{dia}$ para mulheres, não apresenta efeitos prejudiciais à insuficiência cardíaca. Porém, pacientes com IC devem ser orientados no sentido de minimizar a ingestão de bebidas alcoólicas ${ }^{30}$.

\section{Fracionamento e volume}

Para evitar sobrecarga prandial e uma meIhor absorção dos nutrientes é importante oferecer uma dieta com volume reduzido e fracionamento aumentado ( 6 a 8 refeições/dia). A consistência da dieta deve ser modificada em casos de dispnéia, disfagia, odinafagia e dificuldade masti- gatória. Se a ingestão oral estiver abaixo de $60 \%$, a suplementação com fórmula enteral se faz necessária ${ }^{20}$.

\section{Suplementação nutricional}

Ainda não existem pesquisas que estabeleçam as necessidades adequadas de micronutrientes na insuficiência cardíaca e os estudos sobre a suplementação nutricional nesta morbidade, também são escassos e incluem um número reduzido de pacientes. Portanto, ainda não há uma conclusão definitiva quanto aos benefícios da suplementação nutricional, em especial de vitaminas e minerais na IC. Contudo, dois aspectos precisam ser levantados a respeito desse assunto. A maior parte dos estudos com suplementação foca em um único nutriente, desconsiderando a cascata de interações metabólicas que envolvem os micronutrientes e, dessa forma, acarretando em conclusões que não demonstram efetividade ${ }^{32}$. Se não há recomendações específicas para a insuficiência cardíaca, o mais indicado é a utilização da Ingestão Dietética Recomendada (Recommended Dietary Intakes - RDA), que representa um dos níveis de referência da Ingestão Dietética de Referência (Dietary Reference Intakes - DRI), porém, estas foram estabelecidas para atender as necessidades de indivíduos saudáveis e não pessoas enfermas.

Assim sendo, deve-se estar atento para os micronutrientes mais importantes na IC e de acordo as necessidades clínicas do paciente, utilizá-los não apenas com base na RDA, mas considerando também o Limite de Ingestão Máxima Tolerável (Tolerable Upper Intake Level - UL), que representa o mais alto nível de ingestão de um nutriente que não causará efeitos adversos à saúde da maioria das pessoas. Acima da UL, o risco de efeitos adversos aumenta sensivelmente ${ }^{33}$. Essas recomendações, quando possíveis, podem ser obtidas dos próprios alimentos, mas, em alguns casos, a suplementação de vitaminas e minerais, bem como de macronutrientes para complementar o valor energético da dieta, pode ser indicada ${ }^{20}$. 
Um estudo ${ }^{32}$ randomizado, duplo-cego, controlado com 30 indivíduos, avaliou o efeito da suplementação de micronutrientes (Quadro 1) em pacientes com IC. Ao final observou-se melhora do volume e da fração de ejeção do ventrículo esquerdo, bem como da qualidade de vida. Este foi o primeiro ensaio clínico com uso de micronutrientes em pacientes com IC. Apesar das limitações do estudo (tamanho reduzido da amostra, ausência da avaliação dos hábitos alimentares e do estado nutricional dos pacientes) os resultados mostraram que a suplementação de micronutrientes melhora o volume ventricular esquerdo, a fração de ejeção, os níveis de homocisteína e a qualidade de vida, porém não houve modificações no que se refere ao teste de seis minutos e aos níveis de citocinas inflamatórias.

\section{Micronutrientes}

Tiamina: a deficiência de tiamina leva a um prejuízo do metabolismo oxidativo especialmente dos carboidratos, favorecendo um acúmulo de piruvato e lactato, o que pode agravar a insuficiência cardíaca. Vale lembrar que o uso de diuréticos aumenta a excreção de tiamina favorecendo sua depleção ${ }^{34-36}$.

Vitaminas B6, B12 e ácido fólico: estas três vitaminas participam do metabolismo da homocisteína sendo capazes de reduzir os níveis séricos deste aminoácido que apresenta efeitos inotrópicos negativos, provavelmente mediados por efeitos sobre a função endotelial ${ }^{34,35}$

Vitaminas lipossolúveis $(A, D, E$ e $K)$ : quando há má absorção das gorduras estas vitaminas

Quadro 1. Doses de micronutrientes: suplementadas, RDA e UL.

\begin{tabular}{|c|c|c|c|}
\hline Nutriente & Dose diária (quatro cápsulas) & RDA (adultos>50 anos) & UL (adultos $>50$ anos) \\
\hline Cálcio & $250 \mathrm{mg}$ & $1200 \mathrm{mg}$ & $2500 \mathrm{mg}$ \\
\hline \multirow[t]{2}{*}{ Magnésio } & $150 \mathrm{mg}$ & Homens: 420mg & $350 \mathrm{mg}$ \\
\hline & & Mulheres: $320 \mathrm{mg}$ & \\
\hline \multirow[t]{2}{*}{ Zinco } & $15 \mathrm{mg}$ & Homens: $11 \mathrm{mg}$ & $40 \mathrm{mg}$ \\
\hline & & Mulheres: $8 \mathrm{mg}$ & \\
\hline Cobre & $1,2 \mathrm{mg}$ & 900mcg & $10 \mathrm{mg}$ \\
\hline Selênio & $50 \mathrm{mcg}$ & $55 \mathrm{mcg}$ & 400mcg \\
\hline \multirow[t]{2}{*}{ Vitamina A } & $800 \mathrm{mcg}$ & Homens: 900mcg & $3000 \mathrm{mcg}$ \\
\hline & & Mulheres: $700 \mathrm{mcg}$ & \\
\hline \multirow[t]{2}{*}{ Tiamina } & $200 \mathrm{mg}$ & Homens: $1,2 \mathrm{mg}$ & Sem limites \\
\hline & & Mulheres: $1,1 \mathrm{mg}$ & \\
\hline \multirow[t]{2}{*}{ Riboflavina } & $2 \mathrm{mg}$ & Homens: $1,3 \mathrm{mg}$ & Sem limites \\
\hline & & Mulheres: $1,1 \mathrm{mg}$ & \\
\hline \multirow[t]{2}{*}{ Vitamina $B_{6}$} & $200 \mathrm{mg}$ & Homens: $1,7 \mathrm{mg}$ & $100 \mathrm{mg}$ \\
\hline & & Mulheres: $1,5 \mathrm{mg}$ & \\
\hline Folato & $5 \mathrm{mg}$ & 400mcg & Sem limites \\
\hline Vitamina $\mathrm{B}_{12}$ & 200mcg & $2,4 \mathrm{mcg}$ & Sem limites \\
\hline \multirow[t]{2}{*}{ Vitamina C } & $500 \mathrm{mg}$ & Homens: 90mg & $2000 \mathrm{mg}$ \\
\hline & & Mulheres: $75 \mathrm{mg}$ & \\
\hline Vitamina $\mathrm{E}$ & $400 \mathrm{mg}$ & $15 \mathrm{mg}$ & $1000 \mathrm{mg}$ \\
\hline Vitamina D & $10 \mathrm{mcg}$ & Não existe & $50 \mathrm{mcg}$ \\
\hline Coenzima Q10 & $150 \mathrm{mg}$ & Não existe & Não existe \\
\hline
\end{tabular}

RDA: recommended dietary intakes; UL: tolerable upper intake level.

Fonte: Adaptado de Witte et al. ${ }^{3}$ e Committee on the Scientific Evaluation of Dietary Reference Intakes ${ }^{33}$. 
também não são adequadamente absorvidas, sendo necessária a reposição das mesmas ${ }^{36}$.

Coenzima Q10: pacientes com insuficiência cardíaca apresentam baixos níveis de coenzima Q10 no miocárdio. Esta é essencial para o transporte de elétrons na cadeia respiratória mitocondrial, além de funcionar como potente antioxidante ${ }^{35-37}$. Em uma meta-análise ${ }^{38}$ foram investigados os efeitos da coenzima Q10 em pacientes com insuficiência cardíaca congestiva sobre parâmetros cardíacos hemodinâmicos e observou-se que $73 \%$ dos pacientes melhoraram a função cardíaca; $88 \%$ melhoraram o índice de volume diastólico final e $92 \%$ melhoraram a fração de ejeção, concluindo que a coenzima Q10 pode atuar como adjuvante no tratamento da Insuficiência Cardíaca Congestiva (ICC).

Cálcio, magnésio e vitamina $D$ : estes nutrientes são essenciais para a manutenção da massa óssea. Sabe-se que 50\% dos pacientes com insuficiência cardíaca apresentam osteopenia ou osteoporose. Além disso, baixo nível de cálcio é potencialmente proarrítimico e baixos níveis de magnésio são sempre associados ao pobre prognóstico na IC e sua correção melhora a função cardíaca. É importante citar que o uso de diuréticos também aumenta a excreção urinária de cálcio e magnésio ${ }^{36,37}$.

Selênio, zinco, manganês, cobre, vitaminas $B_{2}$ (riboflavina), C e $E$ : todos funcionam como potentes antioxidantes contribuindo para redução do estresse oxidativo e dos danos provocados pelo mesmo ${ }^{34,36,37}$. Os antioxidantes podem ter uma ação direta (vitamina C e E) ou agir como componentes de enzimas antioxidantes, como o zinco, o cobre e o manganês, que participam da estrutura da enzima superóxido dismutase ou o selênio e a vitamina $B_{2}$, essenciais para a síntese de glutationa peroxidase e glutationa redutase, respectivamente. Aceita-se, mas ainda não foi comprovado, que uma dieta com quantidades adequadas de antioxidantes podem prevenir a insuficiência cardíaca. Estudos clínicos e experimentais têm sugerido que a IC pode estar associada ao aumento de radicais livres e aos reduzidos níveis de antioxidantes $^{35}$. A deficiência de selênio tem sido identificada como importante fator etiológico de IC não isquêmica, especialmente em áreas onde o solo é pobre neste mineral, como no leste da China e no oeste da África ${ }^{35}$. Pacientes com IC apresentam níveis plasmáticos de selênio mais baixos que indivíduos saudáveis e os níveis deste mineral estão fortemente relacionados com o consumo máximo de oxigênio e a tolerância ao exercício, mas sem correlação com a função ventricular esquerda ${ }^{35}$.

Taurina: aminoácido com capacidade antioxidante e o mais importante regulador endógeno da homeostase do cálcio intracelular. A deficiência de taurina, por si só, é capaz de produzir cardiomiopatia ${ }^{34}$.

Apesar da importância do estado nutricional no prognóstico do paciente com insuficiência cardíaca e de se saber que estes apresentam uma inadequada ingestão de nutrientes, bem como maior perda e maiores necessidades dos mesmos, ainda existem poucos estudos sobre a adequação da dieta destes indivíduos. O que pode ser observado, após esta revisão, é que a dietoterapia do paciente com IC é muito focada na questão do controle do sal e de líquido, sendo dada pouca atenção à quantidade e à qualidade dos demais nutrientes. Esta revisão permitiu ter uma idéia mais ampla da correta adequação da dieta, com as principais recomendações que se tem até então (Quadro 2).

Para o planejamento da terapia nutricional adequada de um indivíduo com insuficiência cardíaca é necessário: (1) estimar as necessidades e a ingestão alimentar habitual para verificar a existência de desvios quanto à adequação dos nutrientes; (2) realizar a avaliação física dos mesmos, incluindo a determinação do percentual de massa gorda e massa magra; (3) fazer uma adequada avaliação dos sinais e sintomas associados à deficiência, bem como do excesso de nutrientes; (4) utilizar análises bioquímicas que também possam guiar quanto à adequação dos nutrientes ingeridos (5) considerar os sintomas e fisiopatologias da doença, bem como as interações drogas-nutrientes. Dessa forma os erros dietéticos, certamente, serão minimizados e o tratamento não-farmacológico estará otimizado. 
406 | V. SAHADE \& V.S.P. MONTERA

Quadro 2. Adequações dietéticas para pacientes com insuficiência cardíaca.

\begin{tabular}{|c|c|c|}
\hline Nutrientes & Recomendações & Autores \\
\hline Valor energético da dieta & 28 a $32 \mathrm{kcal} / \mathrm{kg}$ & Aquilani et al. ${ }^{18}$ \\
\hline Carboidrato & $\begin{array}{l}50 \text { a } 60 \% \\
\text { Priorizar carboidratos de baixa carga glicêmica }\end{array}$ & $\begin{array}{l}\text { Berry \& } \text { Clarck }^{9} \\
\text { Ross et al. }{ }^{19} \\
\text { Heart Failure Society of America }{ }^{20}\end{array}$ \\
\hline Proteína & 0,8 a $2,0 \mathrm{~g} / \mathrm{kg} / \mathrm{dia}$ & $\begin{array}{l}\text { Quinn et al.\& Askanazi22 } \\
\text { Aquilani et al. }{ }^{18}\end{array}$ \\
\hline $\begin{array}{l}\text { Lipídio } \\
\text { Gordura saturada }\end{array}$ & $\begin{array}{l}<30 \% \\
<7 \%\end{array}$ & $\begin{array}{l}\text { Heymsfield et al. } \\
\text { Gibbs et al. }{ }^{23}\end{array}$ \\
\hline $\begin{array}{l}\text { Gordura mono e poliinsaturada } \\
\text { Gordura trans }\end{array}$ & $\begin{array}{l}\text { enfatizando ácidos graxos da série ômega } 3 \\
<1 \%\end{array}$ & $\begin{array}{l}\text { Tavazzi et al. } .^{24} \\
\text { Heart Failure Society of America }{ }^{20}\end{array}$ \\
\hline Sódio & $2-3 \mathrm{~g} / \mathrm{dia}$ & $\begin{array}{l}\text { Diretriz Latino Americana para Avaliação e } \\
\text { Conduta na IC Descompensada }{ }^{26}\end{array}$ \\
\hline Líquido & Avaliar necessidade de restrição & $\begin{array}{l}\text { Diretriz Latino Americana para Avaliação e } \\
\text { Conduta na IC Descompensada }{ }^{26}\end{array}$ \\
\hline Álcool & $\begin{array}{l}\text { Consumo moderado } \\
\text { Homens:30g/dia } \\
\text { Mulheres: } 20 \mathrm{~g} / \mathrm{dia}\end{array}$ & $\begin{array}{l}\text { Salisbury et al. }{ }^{31} \\
\text { Urbano-Marquez \& Fernadez-Sola }{ }^{30}\end{array}$ \\
\hline Vitaminas e minerais & $\begin{array}{l}\text { De acordo com as necessidades do paciente } \\
\text { Mínimo: RDA } \\
\text { Máximo: UL }\end{array}$ & Witte et al. ${ }^{32}$ \\
\hline Suplementação de vitaminas e minerais & $\begin{array}{l}\text { De acordo com as necessidades do paciente } \\
\text { Mínimo: RDA } \\
\text { Máximo: UL }\end{array}$ & Witte et $a l .32$ \\
\hline
\end{tabular}

\section{O N C L U S Ã O}

A terapia nutricional objetiva a recuperação e/ou a manutenção do estado nutricional do paciente pela educação nutricional e planejamento dietético. É de fundamental importância que o paciente compreenda as razões das modificações alimentares, pois só dessa maneira a aderência irá ocorrer efetivamente. A dieta participa do tratamento da insuficiência cardíaca como importante coadjuvante, favorecendo um melhor prognóstico.

Existe uma crescente evidência de que a dietoterapia é um fator importante no prognóstico e no tratamento dos pacientes com insuficiência cardíaca, porém ainda há diversas lacunas no que diz respeito à suplementação de nutrientes. Dessa maneira, futuros estudos, incluindo ensaios clínicos randomizados, fazem-se necessários para que se possa prescrever, com segurança, doses de nutrientes acima das quantidades recomendadas para esses indivíduos. Portanto, a dietoterapia do paciente com insuficiência cardíaca é muito mais ampla e complexa do que a simples restrição de sódio e líquido.

\section{COLABORADORES}

V. SAHADE planejou o estudo, revisou a bibliografia, preparou a redação do manuscrito. V.S.P. MONTERA revisou a bibliografia e preparou o manuscrito.

\section{REFERÊ NCIAS}

1. American Colege of Cardiology. American Heart Association 2005 guideline update for the diagnosis and management of chronic heart failure in the adult. Circulation. 2005; 112:e154-e235.

2. Stewart S, MacIntyre K, Hole DJ, Capewell S, McMurray JJ. More 'malignant' than cancer? Five-year survival following a first admission for heart failure. Eur J Heart Fail. 2001; 3(3):315-22. 
3. Sharpe N, Doughty R. Epidemiology of heart failure and ventricular dysfunction. Lancet. 1998; 352: (Suppl)l:3-7.

4. II Diretrizes da Sociedade Brasileira de Cardiologia para o diagnóstico e tratamento da insuficiência cardíaca. Arq Bras Cardiol. 2002; 79 (4):1-30.

5. Brasil. Ministério da Saúde. Morbidade hospitalar do SUS. Brasília: Sistema de Informações Hospitalares do SUS; 2003.

6. Witte KA, Clark AL. Chronic heart failure and micronutrients. J Am Coll Cardiol. 2001; 37 (7): 165-74.

7. Heymsfield SB, Smith J, Redd S, Whitworth HB Jr. Nutrition support in cardiac failure. Surg Clin North Am. 1981; 61(6):635-52.

8. Anker SD, Ponikowski P, Varney S. Chua TP, Clark $A L$, Webb-Peploe KM, et al. Wasting as independent risk factor for mortality in chronic heart failure. Lancet. 1997; 349(9058):1050-3.

9. Berry C, Clark AL. Catabolism in chronic heart failure. Eur Heart J. 2000; 21(7)21-32.

10. Obisesan TO, Toth MJ, Donaldson K, Gottlieb SS, Fisher ML, Vaitekevicius $\mathrm{P}$, et al. Energy expenditure and symptom severity in men with heart failure. Am J Cardiol. 1996; 77(14):1250-2.

11. Poehlman ET, Scheffers J, Gottlieb SS, Fischer ML, Vaitekevicius P. Increased resting metabolic rate in patients with congestive heart failure. Ann Arch Intern Med. 1994; 121(11)60-2.

12. King D, Smith ML, Chapman TJ, Stockdale HR, Lye M. Fat malabsorption in elderly patients with cardiac cachexia. Age Ageing.1996; 25(2):144-9.

13. Ajayi AA, Adigun AQ, Ojofeitimi EO, Yusuph H. Anthropometric evaluation of cachexia in chronic congestive heart failure: the role of tricuspid regurgitation. Inter J Cardiol. 1999; 71(1):79-84.

14. Kenchaiah S, Evans JC, Levy D, Wilson PW, Benjamim EJ, Larson MG, et al. Obesity and the risk of heart failure. N Engl J Med. 2002; 347(5):305-13.

15. Horwich TB, Fonarow GC, Hamilton MA, MacLellan WR, Woo MA, Tillisch JH. The relationship between obesity and mortality in patients with heart failure. J Am Coll Cardiol. 2001; 38(3):789-95.

16. Lissin LW, Gauri AJ, Froelicher VF, Ghayoumi A, Myers J, Giacommini J. The prognostic value of body mass index and standart exercise testing in male veterans with congestive heart failure. J Card Fail. 2002; 8(4):206-15.

17. Gustafsson F, Kragelund CB, Torp-Pedersen C. Effect of obesity and being overweight on longterm mortality in congestive heart failure: influence of left ventricular systolic function. Eur Heart J. 2005; 26(1):58-64.

18. Aquilani R, Opasich C, Verri M, Boschi F, Febo O, Pasini $E$, et al. Is nutritional intake adequate in chronic heart failure patients? J Am Coll Cardiol. 2003; 42(7):1218-23.

19. Ross AC, Olson JA, Shils ME. Tratado de nutrição moderna na saúde e na doença. 9a. ed. São Paulo: Manole; 2003.

20. Heart Failure Society of America. Executive summary: HFSA 2006 comprehensive heart failure practice guideline. J Card Fail. 2006; 12(1):10-38.

21. Fibras em nutrição enteral. Publicações Nestlé. [acesso 2006 fev 20]. Disponível em: <http:// www.nestle.com.br/healthcarenutrition>.

22. Quinn T, Askanazi J. Nutrition and cardiac disease. Crit Care Clin. 1987; 3(1):167-84.

23. Gibbs $C R$, Jackson G, Lip GY. ABC of heart failure. Non-drug management. BMJ. 2000; 320(7231): 366-9.

24. Tavazzi L, Tognoni G, Franzosi MG, Latini R, Maggioni AP, Marchioli $R$, et al. Rationale and design of the GISSI heart failure trial: a large trial to assess the effects of n-3 polyunsaturated fatty acids and rosuvastatin in symptomatic congestive heart failure. Eur J Heart Fail. 2004; 6:(5)635-41.

25. He J, Ogden LG, Bazzano LA, Vupputuri S, Loria C, Whelton PK. Dietary sodium intake and incidente of congestive heart failure in overwight US men and woman. Arch Intern Med. 2002; 162(14): 1619-24.

26. Diretriz Latino Americana para avaliação e conduta na insuficiência cardíaca descompensada. Arq Bras Cardiol. 2005; 85(3):12-13.

27. Stevenson LW, Nohria A, Mielniczuk L. Torrent or torment from the tubules? Challenge of cardiorenal connections. J Am Coll Cardiol. 2005; 45(12):2004-7.

28. van der Wal $M H$, Jaarsma $T$, van Veldhuisen DJ. Non compliance in patients with Herat failure: how can we manage it? Eur J Heart Fail. 2005; 7(1):5-17.

29. Evangelista LS, Doering LV, Dracup K. Usefulness aft a history of tobacco and alcohol use in predicting multiple heart failure readmissions among veterans. Am J Cardiol. 2000; 86(12): $1339-42$.

30. Urbano-Marquez A, Fernandez-Sola J. Alcohol consumption and heart failure. J Card Fail. 2005; 11(5):329-32.

31. Salisbury AC, House JA, Conard MW, Krumholz HM, Spertus JA. Low-to-moderate alcohol intake and health status in heart failure patients. J Card Fail. 2005; 11(5):323-8. 
408 | V. SAHADE \& V.S.P. MONTERA

32. Witte KK, Nikitin NP, Parker AC, von Haehling S, Volk HD, Anker SD, et al. The effect of micronutrient supplementation on quality-of-life and left ventricular function in elderly patients with chronic heart failure. Eur Heart J. 2005; 26:(4)2238-44.

33. Committee on the Scientific Evaluation of Dietary Reference Intakes. Dietary reference intakes: the essential reference for dietary planning and assessment. [cited 2007 Nov 11]. Available from: $<$ www.nap.edu>.

34. Sole MJ, Jeejeebhoy KN. Conditioned nutritional requirements: therapeutic relevance to heart failure. Herz. 2002; 27(2):174-8.

35. De Lorgeril M, Salen P, Accominotti M, Cadau M, Steghens JP, Boucher F, et al. Dietary and blood antioxidants in patients with chronic heart failure.
Insights into the potential importance of selenium in heart failure. Eur J Heart Fail. 2001; 3(6):661-9.

36. Witte KK, Clark AL. Nutritional abnormalities contributing to cachexia in chronic illness. Int J Cardiol. 2002; 85(1):23-31.

37. Lorgeril M, Sale P, Defaye P. Importance of nutrition in chronic heart failure patients. Eur Heart J. 2005; 26(4):2215-7.

38. Soja AM, Mortensen SA. Treatment of congestive heart failure with coenzyme Q10 illuminated by meta-analyses clinical trials. Mol Aspects Med. 1997; 18(Suppl 1):S159-S68.

Recebido em: 20/8/2007

Versão final reapresentada em: 27/1/2009 Aprovado em: 30/3/2009 